\title{
Warm brane inflation with an exponential potential: A consistent realization away from the swampland
}

\author{
Vahid Kamali, ${ }^{1,2,3}$ Meysam Motaharfar, ${ }^{4}$ and Rudnei O. Ramos ${ }^{5}$ \\ ${ }^{1}$ Department of Physics, McGill University, Montreal, Quebec, H3A 2T8, Canada \\ ${ }^{2}$ Department of Physics, Bu-Ali Sina (Avicenna) University, Hamedan 65178, 016016, Iran \\ ${ }^{3}$ School of Physics, Institute for Research in Fundamental Sciences (IPM), 19538-33511, Tehran, Iran \\ ${ }^{4}$ Department of Physics, Shahid Beheshti University, G. C., Evin,Tehran 19839, Iran \\ ${ }^{5}$ Departamento de Fúsica Teórica, Universidade do Estado do Rio de Janeiro, 20550-013 Rio de Janeiro, RJ, Brazil
}

\begin{abstract}
It has very recently been realized that coupling branes to higher dimensional quantum gravity theories and considering the consistency of what lives on the branes, one is able to understand whether such theories can belong either to the swampland or to the landscape. In this regard, in the present work, we study a warm inflation model embedded in the Randall-Sundrum braneworld scenario. It is explicitly shown that this model belongs to the landscape by supporting a strong dissipative regime with an inflaton steep exponential potential. The presence of extra dimension effects from the braneworld allow achieving this strong dissipative regime, which is shown to be both theoretically and observationally consistent. In fact, such strong dissipation effects, which decrease towards the end of inflation, together with the extra dimension effect, allow the present realization to simultaneously satisfy all previous restrictions imposed on such a model and to evade the recently proposed swampland conjectures. The present implementation of this model, in terms of an exponential potential for the scalar field, makes it also a possible candidate for describing the late-time Universe in the context of a dissipative quintessential inflation model and we discuss this possibility in the Conclusions.
\end{abstract}

\section{INTRODUCTION}

Flattening, isotropizing, and homogenizing the Universe and reproducing the adiabatic, nearly Gaussian, and quasiscale invariant spectrum of primordial density fluctuations in accordance with the observational cosmological data [1] require implementing a mechanism complementing the standard big bang cosmology to portray the very early Universe. There are today several scenarios attempting to achieve all of these features, namely, ekpyrotic/cyclic theories [2, matter-bounce scenario [3, pre-big bang cosmology [4, string gas cosmology [5], pseudoconformal cosmology 6], to name but a few. Yet, inflation [7], an exponentially accelerated expansion driven by a scalar field $\phi$ rolling down a sufficiently flat region of a potential $V(\phi)$, is still the simplest and most successful one among all other alternatives. Despite its tremendous success, inflation suffers from some long-lasting conceptual problems, namely, the fine-tuning problem [8], the initial condition problem 9], the transPlanckian problem [10, the measure problem [11, singularity problems [12, etc, which makes inflation to be known as a paradigm rather than a well-established theory after three decades.

Cosmologists believe that some, if not all, of the above problems might be solved by consistently embedding an inflation model, taken as an effective field theory of gravity, into a $\mathrm{M} / \mathrm{string}$ theory, taken as a candidate for a quantum theory of gravity. If this construction is possible, then one could in principle build an ultraviolet (UV) complete model for inflation. Although great efforts have been made to accomplish such a goal, there is still no conclusive result for such possibility so far (see, e.g., discussions in Ref. [13]). The most recent efforts led into the so-called swampland conjectures 14, which requests steep potentials $V$, such that $M_{\mathrm{Pl}}\left|V^{\prime}\right| / V \gtrsim \mathcal{O}(1)$, with a sub-Planckian field excursion, $\Delta \phi \lesssim M_{\mathrm{Pl}}$ (for a general review on the swampland conjectures, see, e.g., Ref. [15] and also the references therein for the many implications in cosmology). While the latter condition can be satisfied with some inflationary models $[16$, the former condition given on the potential rules out most slow-roll single field inflationary models, requesting the violation of the slowroll regime.

Among the many recent discussions concerning these conjectures, the authors in Ref. [17] have very recently explained the necessity of the swampland constraints utilizing the completeness of the spectrum of charged branes in a quantum theory of gravity and the consistency of what lives on the branes. In fact, they have shown that coupling branes, as a consistent ingredient of higher dimensional theories, to quantum theories of gravity in higher dimensions, are useful to separate theories which are in the landscape from those which belong to the swampland. Baneworld type of models have been extensively used in cosmology. In particular the braneworld models from Randall and Sundrum [18, which we will focus in the present work, also known as Randall-Sundrum (RS) type I and II models. In such theories, all the standard particles and their interactions are confined to the brane and just gravity can propagate along a fifth dimension. The extra dimension modifies the Friedmann equation on the brane, leading in particular to a quadratic term proportional to the energy density, which have some significant cosmological implications [19]. The scenario of inflation in RS II braneworld was studied in Ref. [20], in which the authors have shown that brane corrections at the high energy regime allow steep potentials to be 
embedded in the braneworld scenario. More recently, the authors in Ref. 21] have also discussed about the swampland in this model.

In the present work, we will focus on an inflation model with an exponential potential in the context of the RS II braneworld scenario. Let us recall that exponential potentials for scalar fields can naturally emerge in $\mathrm{M} /$ string theory from the compactification of the higher dimensions into a four-dimensional effective field theory, that encodes the size of the extra dimensions 22 . It has been shown that an exponential potential form for the scalar inflaton field (and with canonical kinetic term) leads to a power-law type of solutions, with the scale factor satisfying $a(t)=t^{q}$ and $q>1$. This type of potential can also describe different cosmological epochs, including, for instance, the behavior of the late-time Universe [23]. Moreover, it leads to an everlasting power law inflation at the very early Universe, with constant slow-roll parameters [24, 25]. Despite all of this, several attempts have been made towards overcoming such difficulties, like, for instance, implementing these exponential potential models in the braneworld scenario [26 28]. Although inflation with an exponential potential in the braneworld scenario can have a graceful exit, it is still in tension with the observational data. Even in a braneworld scenario, an exponential potential model for inflation produces a large tensor-to-scalar ratio, so it is considered excluded by the observational data 28. It also requires an efficient reheating mechanism [26]. Besides, it suffers from a long kination period after inflation, leading to a large amount of gravitational particle production and relic gravity waves, disrupting and even undermining the big bang nucleosynthesis (BBN) [27.

Although dissipation is an indispensable part of any physical system interacting with its environment, conventional inflationary scenarios, namely cold inflation, typically neglect such effects during the inflationary phase. Thus, a (p)reheating phase at the end of inflation is required so to connect with the big bang radiation dominated regime. In the warm inflation (WI) picture 29] the possible dissipative effects that results from the interactions of the inflaton field with other field degrees of freedom can sustain a subdominant radiation bath throughout the inflationary dynamics. If the dissipative effects can become strong enough, the Universe can smoothly enter into the radiation dominated era, with no separate reheating phase being in general required [30. There is by now an extensive literature on different aspects of WI, the models giving origin to it and on its applications [3148. For instance, the inflaton itself can be a source and responsible for cosmic magnetic field generation [31. In combination with the intrinsic dissipative effects lead to a successful baryogenesis scenario 32. There can also be a remnant inflaton field behaving like dark matter 33 and dark energy [34 36. Furthermore, such dissipation effects change the dynamics of inflation due to the supplementary friction term, making it possible to construct models with steep potentials [37, 38. Moreover, the dissi- pation and radiation effects modify the primordial spectrum of curvature perturbations [39 41, resulting in a lower energy scale of inflation and making it also possible to reconcile steep potentials with the observational data [42, 45]. Such appealing features of WI allow it to simultaneously satisfy the so-called swampland conjectures, provided WI can occur with a sufficient strong dissipation [46, 47]. Furthermore, strong dissipation effects are also able to suppress the energy scale of brane inflation, making it consistent with the observations even in the high energy regime [48. Although it is enormously challenging achieving strong dissipative regimes in WI, a recently proposed new model was built using first principles in quantum field theory and shown explicitly to overcome such difficulties 47.

It is the purpose of the present work to investigate WI in a model with an exponential potential in the RS II braneworld scenario. We are going to explicitly see how it is possible to make this WI model realization fully functional and consistent with both observations and also evading the theoretical restrictions faced by this type of model in its previous constructions done in the context of cold inflation. As an additional welcome feature, we also show that it can support strong enough dissipation to evade the previous, but also the more recent, swampland conjectures.

The paper is organized as follows. In Sec. II, we briefly review the WI dynamics constructed in the RS II braneworld scenario. In Sec. III] we discuss several limitations imposed on the exponential potential considered in this work. We also give the motivations for considering such a potential and discuss how WI is going to remove the discussed previous limitations imposed on the model. In Sec. IV] we give the explicit implementation of the model in WI and demonstrate its viability through explicit numerical analysis. Finally, in Sec. $\mathrm{V}$, we give our conclusions and also discuss the viability of the model to unify the cosmology of the very early Universe with the present epoch through a possible quintessential inflation scenario.

\section{WARM INFLATION DYNAMICS IN THE BRANEWORLD SCENARIO}

In this section, we consider the RS II braneworld scenario in which our four-dimensional world has its space dimensions like a 3 brane embedded in a fivedimensional bulk. In such a braneworld scenario, adopting a Friedmann-Lemaître-Robertson-Walker (FLRW) metric, the cosmological evolution can be described by a generalized Friedmann equation as follows [20, 49]

$$
H^{2} \equiv\left(\frac{\dot{a}}{a}\right)^{2}=\frac{1}{3 M_{\mathrm{Pl}}^{2}} \rho+\frac{1}{36 M_{5}^{6}} \rho^{2}+\frac{\Lambda_{4}}{3}+\frac{\mu}{a^{4}},
$$

where $a(t)$ is the usual scale factor, $\rho$ is the total energy density in the three-dimensional space, $M_{\mathrm{Pl}} \equiv$ 
$1 / \sqrt{8 \pi G} \simeq 2.4 \times 10^{18} \mathrm{GeV}$ is the reduced Planck mass defined in terms of the usual Newton gravitational constant $G, \Lambda_{4}$ is an effective cosmological constant, $\mu$ is an integration constant which behaves as dark radiation term, and $M_{5}$ is the five-dimensional Planck mass, defined as

$$
M_{5}^{6}=\frac{\lambda M_{\mathrm{Pl}}^{2}}{6}
$$

where $\lambda$ is the intrinsic tension on the brane. The cosmological constant term in Eq. 2.1) can be tuned to zero by an appropriate choice of the brane tension and bulk cosmological constant. Also, the dark radiation term quickly redshifts during inflation so we can also neglect it. Thus, Eq. 2.1 can then be expressed simply as

$$
H^{2}=\frac{1}{3 M_{\mathrm{Pl}}^{2}} \rho\left(1+\frac{\rho}{2 \lambda}\right) .
$$

In the WI scenario, the total energy density is given by

$$
\rho=\frac{\dot{\phi}^{2}}{2}+V(\phi)+\rho_{R},
$$

which accounts for the radiation fluid, with energy density $\rho_{R}$, and the scalar field (the inflaton) $\phi$, with potential $V(\phi)$. The background dynamics for the inflaton field $\phi$ and the radiation energy density $\rho_{R}$, which are coupled to each other, are, respectively, given by

$$
\begin{aligned}
& \ddot{\phi}+3(1+Q) H \dot{\phi}+V_{, \phi}=0 \\
& \dot{\rho}_{R}+4 H \rho_{R}=3 H Q \dot{\phi}^{2}
\end{aligned}
$$

where dots denote temporal derivatives and $Q$ is the dissipation ratio in WI, defined as

$$
Q=\frac{\Upsilon(T, \phi)}{3 H},
$$

where $\Upsilon(T, \phi)$ is the dissipation coefficient in WI, which can be a function of the temperature and/or the background inflaton field, depending on the specifics of the microscopic physics behind the construction of WI. For a radiation bath of relativistic particles, the radiation energy density is given by $\rho_{R}=\pi^{2} g_{*} T^{4} / 30$, where $g_{*}$ is the effective number of light degrees of freedom $\left(g_{*}\right.$ is fixed according to the dissipation regime and interactions form used in WI).

A typical dissipation coefficient functional form in terms of the temperature and the inflaton field amplitude found in many WI models can be expressed as 37, 38, 50, 51,

$$
\Upsilon(T, \phi)=C T^{c} \phi^{p} M^{1-c-p},
$$

where $C$ is a dimensionless constant (that carries the details of the microscopic model used to derive the dissipation coefficient, e.g., the different coupling constants of the model), $M$ is a mass scale in the model and depends of its construction, while $c$ and $p$ are numerical powers, which can be either positive or negative numbers (the dimensionality of the dissipation coefficient in Eq. (2.8) is of course preserved, i.e., $[\Upsilon]=[$ energy $]$ ). Some typical dependencies found in the literature, are the cases with $c=-1, p=2$ (see Ref. [52]), $c=1, p=0$ (see Ref. 45]), $c=-1, p=0$ (in the asymptotic high temperature limit for the dissipation coefficient found in the model of Ref. [47]) and $c=3, p=-2$ (see Refs. [37, 50]). The case with $c=3, p=-2$ was in fact the first microscopic construction for WI from a particle physics model building perspective and giving consistent observables [38]. In the present work we will not be interested in the specific origin of these dissipation coefficients from a microscopic quantum field theory derivation (for such details we refer the interested reader to the above cited references), but on the phenomenological consequences of them.

In terms of Eqs. 2.3, 2.5 and 2.6 the slow-roll parameters in the braneworld scenario are given by

$$
\begin{aligned}
& \epsilon_{\text {brane }}=\epsilon_{V} \frac{1+V / \lambda}{[1+V /(2 \lambda)]^{2}}, \\
& \eta_{\text {brane }}=\eta_{V} \frac{1}{1+V /(2 \lambda)},
\end{aligned}
$$

where $\epsilon_{V}$ and $\eta_{V}$ are the usual slow-roll inflaton potential parameters, defined as

$$
\begin{aligned}
\epsilon_{V} & =\frac{M_{\mathrm{Pl}}^{2}}{2}\left(\frac{V_{, \phi}}{V}\right)^{2}, \\
\eta_{V} & =M_{\mathrm{Pl}}^{2} \frac{V_{, \phi \phi}}{V} .
\end{aligned}
$$

In terms of Eqs. 2.9 and 2.10), the slow-roll conditions in WI are defined by the requirement that $\epsilon_{\text {brane }} \ll 1+Q$ and $\eta_{\text {brane }} \ll 1+Q$. The accelerated inflationary dynamics terminates when $\epsilon_{\text {brane }}=1+Q$.

By assuming the slow-roll approximation, with energy density $\rho \sim V$, the Eqs. 2.3, 2.5 and (2.6) reduce to

$$
\begin{aligned}
& H^{2} \simeq \frac{1}{3 M_{\mathrm{Pl}}^{2}} V\left(1+\frac{V}{2 \lambda}\right), \\
& 3(1+Q) H \dot{\phi} \simeq-V_{, \phi}, \\
& 4 \rho_{R} \simeq 3 Q \dot{\phi}^{2} .
\end{aligned}
$$

Given the dissipation coefficient with the functional form given by Eq. (2.8) and expressing the evolution in terms of the number of e-folds, $d N=H d t$, we can deduce how the dissipation ratio $Q$ and the ratio of temperature of the radiation bath by the Hubble rate, $T / H$, evolve during WI. After some straightforward algebra, we find that their evolution are determined by the equations 


$$
\begin{aligned}
\frac{d \ln Q}{d N} & =\frac{2\left[(2+c) \epsilon_{\text {brane }}-c \eta_{\text {brane }}-2 p \kappa_{\text {brane }}\right]}{4-c+(4+c) Q}, \\
\frac{d \ln (T / H)}{d N} & =\frac{[7+c(Q-1)+5 Q] \epsilon_{\text {brane }}-2(1+Q) \eta_{\text {brane }}+(Q-1) p \kappa_{\text {brane }}}{(1+Q)[4-c+(4+c) Q]}
\end{aligned}
$$

where $\kappa_{\text {brane }}$ is defined as

$$
\kappa_{\text {brane }}=M_{\mathrm{Pl}}^{2} \frac{V_{, \phi}}{\phi V[1+V /(2 \lambda)]} .
$$

Moreover, the ratio of radiation to inflaton energy density in the slow-roll regime is roughly given by

$$
\frac{\rho_{R}}{\rho_{\phi}} \approx \frac{1}{2} \frac{\epsilon_{\text {brane }}}{1+Q} \frac{Q}{1+Q} .
$$

Although the ratio of the radiation to inflaton energy density is very small at the beginning of WI, it can be large at the end of inflation (when $\epsilon_{\text {brane }}=1+Q$ ), even for an initially small dissipation ratio $Q$. Consequently, the Universe can smoothly enter into the radiation dominated epoch at the end of WI, with no need for a separate reheating phase a priori.

\section{THE MODEL}

As explained in the Introduction, in this paper we will be working with the exponential potential form for the inflaton field,

$$
V(\phi)=V_{0} \exp \left(-\alpha \phi / M_{\mathrm{Pl}}\right),
$$

where $V_{0}$ is the normalization for the potential and which can be fixed by the amplitude of primordial curvature perturbations as usual. The study of inflation with this potential is diverse, specially motivated by the fact that a potential like Eq. (3.1) can also serve as a quintessential inflation model, describing both the inflationary early Universe period, as also the late Universe, working as an evolving dark energy model at the present epoch [26]. The exponential potential for the inflaton has also already been studied before in the presence of dissipative effects from particle production [25], yet with no connection to the WI scenario, as it is the focus in the present study.

Despite the many uses of the potential Eq. (3.1), it is, however, hard to find consistent ways of ending inflation in such models in the traditional ways (i.e., ending the acceleration regime). For instance, with the standard slowroll parameters given by Eqs. 2.11) and 2.12), the accelerated expansion requires $\alpha<\sqrt{2}$. However, this leads to a power-law type of inflation, which has long been found to be inconsistent with the observations [53, 54, unless there are either modifications to the form of the potential, to the dynamics, or to both. In the braneworld scenario, it is possible to have inflation even when $\alpha>\sqrt{2}$, provided that the dynamics is dominated by the high energy brane regime, $V>2 \lambda$. In this case, the modification of the dynamics because of the brane corrections are strong and we also have a stronger Hubble friction. As the inflaton field evolves and $V<2 \lambda$, the effects of the brane corrections lessen, the usual general relativity (GR) dynamics is restored, and inflation ends. But the inflaton evolution in a steep potential like Eq. (3.1) will at some point be dominated by the kinetic energy of the field, which gives start to a kination regime. During this regime, the energy density falls off like stiff matter, $\rho \propto 1 / a^{6}$. The abrupt change in the dynamics can produce a large amount of gravitational particle production that might disrupt BBN later on [26]. Furthermore, a large amount of relic gravity waves is also predicted to be generated [27]. The energy density on these produced gravitational waves can be boosted by the kination period and can also disrupt the BBN later on. As an additional problem, the model leads yet to predictions on the tensor-to-scalar ratio and on the spectral tilt of the primordial scalar spectrum that is also excluded by the observations [28. All these issues together have rendered this inflaton potential model unsuitable from an observational point of view.

In the following, we discuss the choice for the dissipation coefficient used in the present study. We will also discuss the theoretical and observational constraints on the parameters of the braneworld scenario and on the exponential potential. Then, we explain under which conditions the WI scenario realization of the model may allow it to overcome all of the above mentioned difficulties, and we then give an explicit example in Sec. IV.

\section{A. Warm inflation implementation}

Considering the model given by Eq. (3.1) in the context of WI, we find, in particular, that the evolution equation for the dissipation ratio Eq. 2.16 becomes

$$
\frac{d \ln Q}{d N}=\frac{4 \lambda \alpha\left\{2 p(2 \lambda+V)+[(2-c) \lambda+2 V] \alpha \phi / M_{\mathrm{Pl}}\right\}}{[4-c+(4+c) Q](2 \lambda+V)^{2} \phi / M_{\mathrm{Pl}}} .
$$


We are interested in the strong dissipative regime of WI, i.e., $Q \gg 1$, which ensures that the swampland conditions are satisfied [46, with the inflaton excursion typically sub-Planckian, $\Delta \phi<M_{\mathrm{Pl}}$. Furthermore, we have the constraint to be discussed below and given by Eq. (3.6), on the inflaton potential constant $\alpha$, requiring rather large values. At the same time, we do not want the dissipation ratio to grow throughout the evolution; otherwise, even by arranging inflation to end due to the braneworld effects, it might reinitiate again or even never end in the first place. Hence, we would ideally want a $d e-$ creasing $Q$ with the number of e-folds, i.e., the right-hand side of Eq. (3.2) should become negative, soon after the braneworld effects become subdominant. Thus, we need to analyze the region of parameters for which the righthand side of Eq. (3.2) can be either positive or negative. In particular, from previous studies on the stability of the WI dynamics [55], we have that the power $c$ should satisfy $-4<c<4$, which results in the denominator of Eq. 3.2 to be always positive. Thus, for $2<c<4$ and $-3<p<0$, we find that the numerator in the righthand side of Eq. (3.2) can be positive in the high energy regime, $V \gg 2 \lambda$, but then it becomes negative as one enters in the low energy regime, $V<2 \lambda$. This is, in particular, exactly the behavior expected with a dissipation coefficient with a functional form with a cubic power in the temperature, as found, e.g., in Refs. [37, 50]. In this case, we find that $Q$ will grow initially during the inflationary evolution, provided that $V>2 \lambda$, decreasing later on in the evolution when $V<2 \lambda$. Thus, by having initially that $\epsilon_{\text {brane }} /(1+Q) \ll 1$, this will ensure that the accelerated expansion will happen both because of the brane effect and because of a growing $Q$, further facilitating the accelerated expansion, even if $\alpha$ is large. On the other hand, as the brane effects lessen and we recover the usual general relativity evolution, the dissipation ratio will start to decrease, eventually ending the accelerated inflationary regime due to the large value of $\alpha$. Note that this effect of the dissipation on the evolution does not happen when $c=1$, as in the case of the dissipation coefficient found in Ref. [45], or in the case of the dissipation coefficient recently found and correspondingly, WI dynamics studied in Ref. [47, which favors a dissipation coefficient with $c=-1$. In both of these two cases, $Q$ will always increase, in general, making it more difficult to end inflation. In fact, such decreasing $Q$ allowed by a $\Upsilon \propto T^{3} / \phi^{2}$ dissipation coefficient not only plays a pivotal role in bringing the inflation to an end but also it will be shown to allow the model studied here to be consistent with the observations, even for large values of the dissipation ratio $Q$. Thus, in the following, we will restrict our study to the cubic in the temperature dissipation coefficient form, wher ${ }^{1}$

$$
\Upsilon(T, \phi)=C \frac{T^{3}}{\phi^{2}} .
$$

In the next section, we will then show our explicit and detailed results for the WI model with the dissipation coefficient Eq. (3.3) in the context of the braneworld scenario. But before entering in the explicit results derived from this model, let us discuss some important constraints that the model might be subjected.

\section{B. Constraints}

In the braneworld scenario, the quadratic term of the energy density in the Hubble parameter Eq. (2.3) becomes dominant in the high energy regime, provided the brane tension $\lambda$ has a moderate value. However, BBN implies that it has to be subdominant, since it decays as $a^{-8}$ and becomes rapidly negligible thereafter. Therefore, the BBN bounds put a lower limit on the brane tension such that it has to satisfy $\lambda \gtrsim(1 \mathrm{MeV})^{4}$, which in combination with Eq. 22.2 gives [20]

$$
M_{5} \gtrsim\left(\frac{1 \mathrm{MeV}}{M_{\mathrm{Pl}}}\right)^{2 / 3} \sim 10 \mathrm{TeV} .
$$

However, considering the fifth dimension to be infinite and requiring relative corrections to the Newtonian law of gravity to be also small [56], one obtains a more stringent constraint as given by $M_{5} \gtrsim 10^{5} \mathrm{TeV}$, or, equivalently, $\lambda \gtrsim 100 \mathrm{GeV}$.

As discussed in Refs. 57, 58, the abundance of gravitinos is related to the reheating temperature through the Boltzmann equation. Hence, by constraining the gravitinos abundance, one can obtain an upper bound on the reheating temperature as $T_{R} \lesssim 10^{6}-10^{8} \mathrm{GeV}$. However, the authors in Ref. [58] have shown that the relation between gravitinos abundance and the reheating temperature breaks in the high energy limit, $V /(2 \lambda)>1$, and allows inflation to occur even with a higher reheating temperature.

The late-time behavior for steep exponential potentials produces a scaling solution, where the scalar field exhibits the same redshift dependence as the dominant fluid in the Universe. Thus, the energy density fraction

\footnotetext{
1 The attentive reader may question what happens with this dissipation coefficient with the exponential potential we use here and where $\phi$ might cross zero. This is not a problem since, as shown in Refs. 37, 38, 50, 51, this dissipation coefficient is derived when the inflaton field is coupled to heavy intermediate fields $\chi$, where the mass $m_{\chi} \propto \phi$, but we could as well have a nonvanishing bare mass for $\chi$, which regulates any possible infrared divergence and avoids $\Upsilon$ to diverge when $\phi \rightarrow 0$.
} 
for the scalar field, assuming spatial flatness, has a scaling solution given by $[23,26]$

$$
\Omega_{\phi}=\frac{3(w+1)}{\alpha^{2}},
$$

where $w$ is the equation of state of the dominant fluid. If the inflaton potential Eq. (3.1) remains unchanged till late in the Universe, it will act like a quintessence field. Early dark energy can influence the cosmic microwave background ( $\mathrm{CMB})$ peaks among to other changes in the CMB power spectrum. Hence, its fraction can be strongly constrained when including small-scale measurements and CMB lensing, for instance. The strongest constraint on the fraction of dark energy at last scattering time has been produced by the Planck data and leading to an upper bound 59, $\Omega_{\phi} \lesssim 0.0036$ at $95 \%$ confidence level (for Planck TT,TE,EE-lowP+BSH). Applying this to Eq. (3.5), we are lead to the lower bound on the coefficient $\alpha$ for the inflaton potential,

$$
\alpha \gtrsim 33.3 \text {. }
$$

Furthermore, the recently proposed swampland conjectures restrict both the dynamics and potential form of the inflationary models [14, 15. In fact, the de Sitter swampland conjecture requests steep potentials, $M_{\mathrm{Pl}} V^{\prime} / V \geq \mathcal{O}(1)$, with no extrema, as a result of which not only inflationary models but also the usual reheating mechanism, due to an oscillatory phase around the minimum at the end of inflation, are ruled out 60. Moreover, the distance swampland conjecture requests subPlanckian field excursions during inflation, $\Delta \phi \lesssim M_{\mathrm{Pl}}$, whereby all large field inflationary models are excluded when the conjecture is taken at face value. As discussed in Ref. [4], to have a large slow-roll parameter $\epsilon_{V}$, one needs to violate the relation between $\epsilon_{H} \equiv-\dot{H} / H^{2}$ and $\epsilon_{V}$ in the conventional single field slow-roll inflation. Besides, one also needs to find another mechanism to heat up the Universe at the end of inflation. The WI scenario has the appealing feature to give us both of these in a single frame due to the presence of the intrinsic dissipation effects, provided that WI occurs in the strong dissipative regime [46, $Q \gg 1$. Moreover, the WI scenario also gives us the possibility of suppressing the tensor-toscalar ratio by several orders of magnitude for a large dissipation ratio, due to effects of the dissipation on the primordial perturbations and the change in the scalar curvature spectrum (to be discussed below). As a result, the inflaton field can remain sub-Plankian during inflation, evading the Lyth bound even for steep potentials. Therefore, dissipation effects make WI able to satisfy the swampland conjectures, while also allowing for the accelerated expansion even when $\alpha>1$ in the exponential potential and braneworld scenario as considered here.

In our numerical study to be presented in the next section, we will keep in mind all of the above constraints and conditions and aim at satisfying them all.

\section{NUMERICAL RESULTS}

As previously mentioned, both the background dynamics and the perturbations get modified due to the presence of dissipation and a radiation bath during WI [39, 40]. As a matter of fact, the primordial power spectrum for WI at horizon crossing can be expressed in the form (see, e.g., Ref. [41] and references therein),

$$
\Delta_{\mathcal{R}}\left(k / k_{*}\right)=\left(\frac{H_{*}^{2}}{2 \pi \dot{\phi}_{*}}\right)^{2} \mathcal{F}\left(k / k_{*}\right)
$$

where the subindex " $*$ " stands for those quantities evaluated at the Hubble radius crossing, $k_{*}=a_{*} H_{*}$. The function $\mathcal{F}\left(k / k_{*}\right)$ in Eq. 4.1) is given by

$$
\mathcal{F}\left(k / k_{*}\right) \equiv\left(1+2 n_{*}+\frac{2 \sqrt{3} \pi Q_{*}}{\sqrt{3+4 \pi Q_{*}}} \frac{T_{*}}{H_{*}}\right) G\left(Q_{*}\right),
$$

where $n_{*}$ denotes the inflaton statistical distribution due to the presence of the radiation bath and $G\left(Q_{*}\right)$ accounts for the effect of the coupling of the inflaton fluctuations to radiation [40]. $G\left(Q_{*}\right)$, in general, can only be determined by numerically solving the full set of perturbation equations in WI and fitting it to an appropriate function. Following an analogous derivation as considered, e.g., in the papers in Ref. [0, we find that an appropriate functional form for $G\left(Q_{\star}\right)$ that is valid for the present exponential model in the braneworld construction and with dissipation coefficient (3.3), is well described by

$$
\begin{aligned}
G\left(Q_{*}\right) & =\frac{1+0.413 Q_{*}^{0.85}}{\left(1+0.18 Q_{*}^{0.859}\right)^{26.5}} \\
& +\frac{0.00692 \exp \left(6.44 Q_{*}^{0.292}\right)}{1+0.00082 \exp \left(0.1763 Q_{*}^{0.66}\right)} .
\end{aligned}
$$

The above equation for $G\left(Q_{*}\right)$ is found to hold for rather very large values for $Q_{*}$, up to around $Q_{*} \simeq 2000$. Besides, since the behavior of the spectrum with $Q_{*}$ is smooth and well behaved, we can always do this procedure (numerical fitting) with a sufficient precision such that any arbitrariness in the numerical fitting does not change the observable quantities, e.g., the spectral index $n_{s}$ and the tensor-to-scalar ratio $r$, both defined below.

In our numerical results, we fix the scalar spectral amplitude value at the pivot scale $k_{*}$ as $\ln \left(10^{10} \Delta_{\mathcal{R}}\right) \simeq$ 3.047, according to the Planck Collaboration 1 1 (in the TT,TE,EE-lowE+lensing + BAO $68 \%$ limits data set).

While the primordial scalar curvature perturbation in WI gets modified according to Eq. 4.1, the tensor perturbations spectrum is unchanged in WI because of the weakness of the gravitational interactions 2 However,

\footnotetext{
${ }^{2}$ See, however, Ref. 61] for possible changes in the tensor spectrum due to WI. However, even for the very large values of $Q_{*}$ considered here by us, those corrections found in Ref. [61] are completely negligible and can be safely neglected.
} 
the spectrum of tensor perturbations is modified in the braneworld scenario due to the presence of the extra dimension, where the graviton resides, with respect to the standard four-dimensional (GR) Universe. The tensor perturbations power spectrum in the braneworld scenario has been determined to be given by 62

$$
\Delta_{T}=\frac{2 H^{2}}{\pi^{2} M_{\mathrm{Pl}}^{2}} F^{2}(x),
$$

where

$$
F(x)=\left[\sqrt{1+x^{2}}-x^{2} \ln \left(\frac{1}{x}+\sqrt{1+\frac{1}{x^{2}}}\right)\right]^{-1 / 2},
$$

with $x=\sqrt{6 M_{\mathrm{Pl}}^{2} H^{2} / \lambda}$.

From Eqs. 4.1) and 4.4, the tensor-to-scalar ratio $r$ is defined as usual,

$$
r=\frac{\Delta_{T}}{\Delta_{\mathcal{R}}}
$$

while the spectral tilt $n_{s}$ is defined as

$$
n_{s}-1=\lim _{k \rightarrow k_{*}} \frac{d \ln \Delta_{\mathcal{R}}\left(k / k_{*}\right)}{d \ln \left(k / k_{*}\right)} .
$$

We recall that the recent data from the Planck Collaboration 63] has placed the upper bound $r<0.056(95 \%$ C.L., Planck TT,TE,EE+lowE+lensing+BK15, at the pivot scale $\left.k_{p}=0.002 / \mathrm{Mpc}\right)$, while for the spectral tilt the result is $n_{s}=0.9658 \pm 0.0040$ (95\% C.L., Planck TT, TE,EE+lowE+lensing+BK15+BAO+running).

In the results shown below, the number of e-folds for inflation, in particular, the number of e-folds before Hubble radius crossing, $N_{*}$, is always computed self-consistently. This is done by noticing that length scales crossing the Hubble radius during inflation and reentering today will satisfy $k_{*}=a_{i} H_{i}=a_{0} H_{0}$, such that 64

$$
\frac{k_{*}}{a_{0} H_{0}}=e^{-N_{*}} \frac{T_{0}}{T_{\text {end }}} \frac{H_{i}}{H_{0}},
$$

where 0 -index quantities mean that they are evaluated today, while $i$-index quantities are those evaluated at $N_{*}$ e-folds before the end of inflation. The CMB temperature today, $T_{0}$, is set to the value $T_{0}=2.725 \mathrm{~K}=$ $2.349 \times 10^{-13} \mathrm{GeV}$. In our convention, we use $a_{0}=1$ and for the Hubble parameter today, we assume the Planck result, $H_{0}=67.66 \mathrm{~km} \mathrm{~s}^{-1} \mathrm{Mpc}^{-1}$ [from the Planck Collaboration [1, TT,TE,EE-lowE+lensing+BAO $68 \%$ limits, $\left.H_{0}=(67.66 \pm 0.42) \mathrm{km} \mathrm{s}^{-1} \mathrm{Mpc}^{-1}\right] . T_{\text {end }}$ is the temperature at the beginning of the radiation dominated regime, which in the WI, turns out to be simply the temperature at the end of inflation, since WI ends by the time the radiation energy density takes over the inflaton one. For the exponential potential Eq. 3.1 in the cold inflation case and in the braneworld scenario, there is a prediction for $N_{*}$ given by [27] $N_{*} \simeq 70$. In the WI case studied here, we always find a smaller value for $N_{*}$ due to the effect of dissipation.

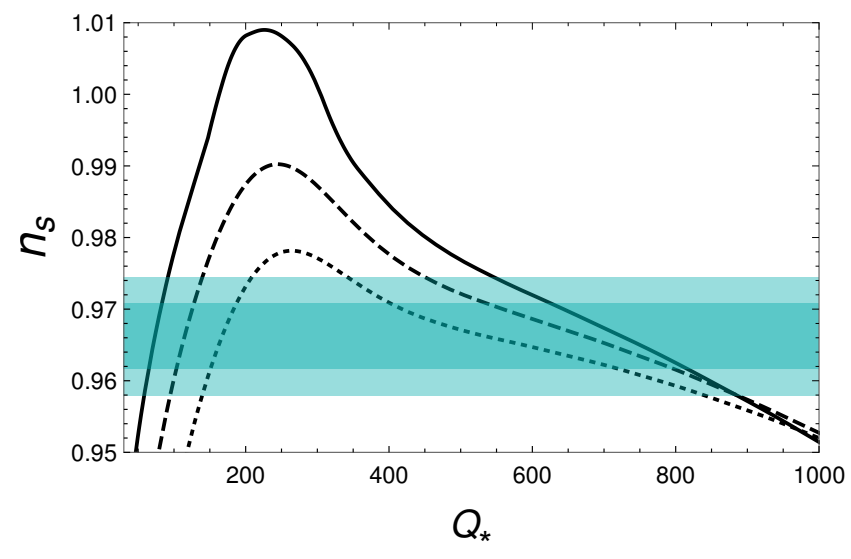

FIG. 1. The spectral index $n_{s}$ as a function of the dissipation ratio $Q_{*}$ for the cases of $\alpha=30$ (solid line), $\alpha=40$ (dashed line), and $\alpha=50$ (dotted line), for a fixed value of (normalized) brane tension, $\lambda / V_{0}=5 \times 10^{-5}$. The shaded areas are for the $68 \%$ and $95 \%$ C.L. results from Planck 2018 $(\mathrm{TT}+\mathrm{TE}+\mathrm{EE}+$ lowE+ lensing $+\mathrm{BK} 15+\mathrm{BAO}$ data $)$.

In Fig. 1, we show the behavior of the spectral index $n_{s}$ as a function of the dissipation ratio $Q_{*}$ at a Hubble radius crossing, when keeping the ratio of the brane tension by the normalization of the inflaton potential, $\lambda / V_{0}$, fixed but for three different values for the exponent $\alpha$ in the inflaton potential. For a strong dissipation ratio, $Q_{*} \gg 1$, the tensor-to-scalar ratio is always quite very small, $r<10^{-14}$. We recall that such very small values for $r$ are typical for WI in the strong dissipative regime. This is so because the scalar curvature power spectrum Eq. 4.1) is completely dominated by the dissipation, thus strongly suppressing the tensor-to-scalar ratio. Whereby, when $Q_{*} \gg 1$, the inflaton statistical distribution term $n_{*}$ in Eq. 4.1 also has a negligible effect, and we can set it as vanishing. From Fig. 1, we see that the larger is the $\alpha$, the steeper is the inflaton potential, therefore, we can find a larger range of dissipation values for which $n_{s}$ agrees with the observational data. We also see that there are always two ranges of $Q_{*}$ values satisfying the observations.

In Fig. 2, we show the behavior of the spectral index $n_{s}$ as a function of the dissipation ratio $Q_{*}$ at the Hubble radius crossing, where now we keep the constant $\alpha$ in the exponential inflaton potential fixed and give the results for three different values for the ratio of the brane tension by the normalization of the inflaton potential. We have a similar behavior as seen in Fig. 11 when considering $\lambda / V_{0}$ fixed. However, the larger is $\lambda / V_{0}$, the smaller is the range of dissipation ratios satisfying the observations. In particular, for $\alpha=40$, we have obtained that when $\lambda / V_{0} \gtrsim 10^{-3}$ there are no longer values for $n_{s}$ found to be compatible with the Planck data, with $n_{s}$ being too red tilted. 


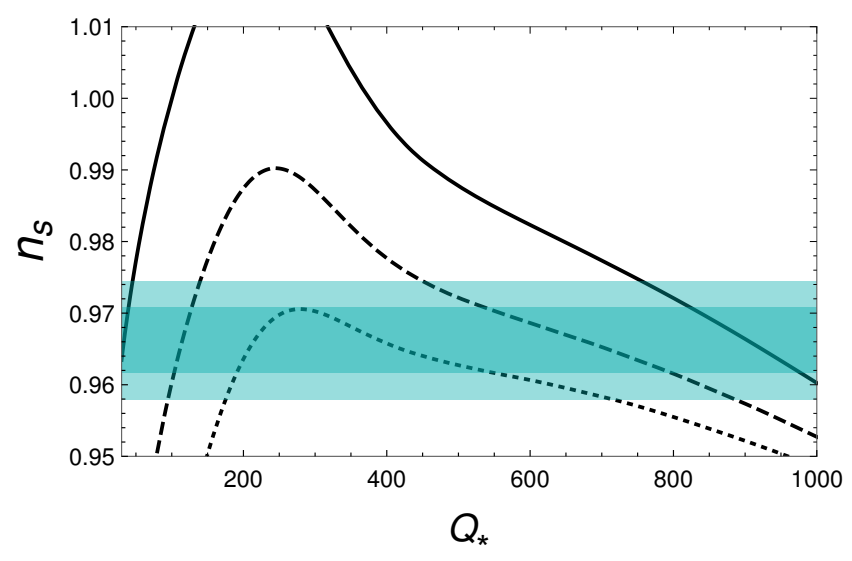

FIG. 2. The spectral index $n_{s}$ as a function of the dissipation ratio $Q_{*}$ for the cases of $\lambda / V_{0}=10^{-5}$ (solid line), $\lambda / V_{0}=5 \times 10^{-5}$ (dashed line) and $\lambda / V_{0}=10^{-4}$ (dotted line), for the fixed value of $\alpha=40$. The shaded areas are for the $68 \%$ and $95 \%$ C.L. results from Planck 2018 $(\mathrm{TT}+\mathrm{TE}+\mathrm{EE}+$ lowE+ lensing $+\mathrm{BK} 15+\mathrm{BAO}$ data $)$.

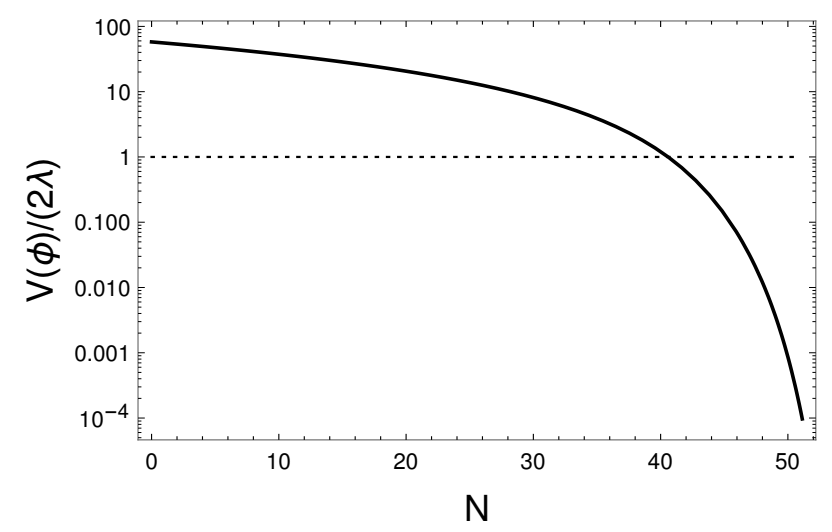

(a)

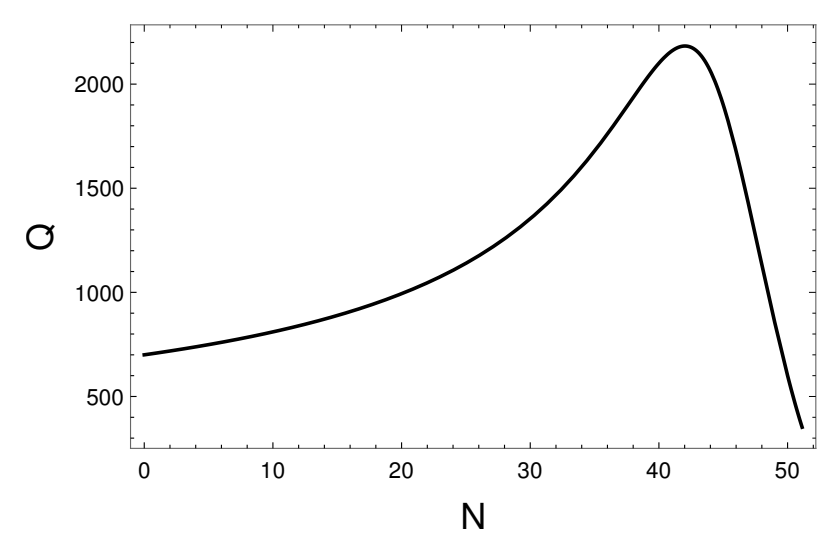

(b)

FIG. 3. The evolution of the ratio $V(\phi) /(2 \lambda)$ [panel (a)] and the dissipation ratio $Q$ [panel (b)], with the number of e-folds, for the choice of parameters $\alpha=40$ and $\lambda / V_{0}=5 \times 10^{-5}$.
In Fig. 3(a), we show the evolution of the ratio $V(\phi) /(2 \lambda)$ with the number of e-folds, while in Fig. 3(b) we show the evolution of the dissipation ratio with the number of e-folds. We have chosen the particular case of $\alpha=40$ and $\lambda / V_{0}=5 \times 10^{-5}$, but there is little change in the results when considering other values of parameters close to these. This is found to be true for all the background quantities in general. From these results we can confirm the behavior for the dissipation ratio with the cubic dissipation coefficient anticipated in the previous section. In the high energy regime, $V>2 \lambda$, i.e., when the brane corrections prevail, $Q$ grows with the the number of e-folds, while when the brane corrections lessen, $V<2 \lambda, Q$ fast decreases.

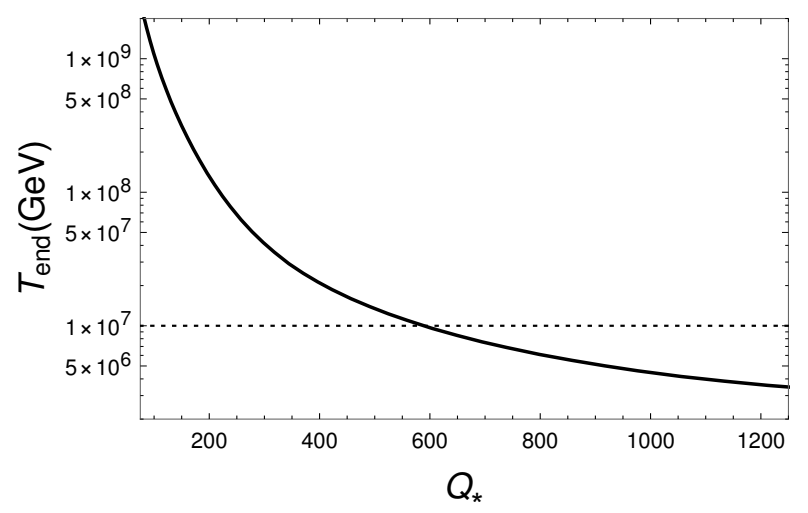

FIG. 4. The temperature at the end of inflation as a function of the dissipation ratio $Q_{*}$ for $\alpha=40$ and $\lambda / V_{0}=5 \times 10^{-5}$.

Considering the same parameters as those used in the previous figure, in Fig. 4 we show the behavior of the temperature at the end of inflation as a function of the dissipation ratio. The thin horizontal dotted line at $T=10^{7} \mathrm{GeV}$ has been drawn as reference only. From the results shown in Fig. 4 , we see that the gravitino bound can comfortably be satisfied for a sufficient large dissipation and the result is also consistent with the Planck data, as seen from Figs. 1 and 2 .

We note that by taking as an explicit example for illustration purposes, the choice of parameters $\alpha=40$, $\lambda / V_{0}=5 \times 10^{-5}$, and $Q_{*}=700$, we find that $n_{s} \simeq 0.965$; that is well within the central value obtained from the Planck legacy data. Furthermore, as expected for such a large dissipation ratio, we have an extremely small tensor-to-scalar ratio, $r \simeq 1.3 \times 10^{-27}$. We also find for these parameter values that the inflaton potential normalization is $V_{0}^{\frac{1}{4}} \simeq 2.219 \times 10^{9} \mathrm{GeV}$ and $N_{*} \simeq 51.1$. For these parameters, we also find that $V_{*}^{1 / 4} \simeq 6.12 \times 10^{8}$ $\mathrm{GeV}$ and that at the end of the inflationary regime, $V_{\text {end }} \simeq 2.20 \times 10^{7} \mathrm{GeV}$. Furthermore, we find that $T_{\text {end }} \simeq 7 \times 10^{6} \mathrm{GeV}$ at the end of inflation. The same parameters produce a sub-Planckian inflaton field excursion, found to be $\Delta \phi \simeq 0.3 \mathrm{M}_{\mathrm{Pl}}$. In addition, the inflaton potential slow-roll parameter, $\epsilon_{V}$, can be larger than 
one, as a consequence of the large value of the dissipation coefficient, while still allowing accelerated expansion by having $\epsilon_{H} \equiv-\dot{H} / H^{2} \ll 1$. These results alone already ensure that the distance and the de Sitter (along also with the refined) swampland conjectures [14, 46] are satisfied. In addition, given the lower energy scale for inflation allowed for the present model in the large dissipation regime, we also find the recently proposed transPlanckian censorship conjecture (TCC) 65] is also satisfied here ${ }^{3}$. Hence, to find that the results found here can satisfy all previously mentioned constraints is quite a pleasant feature 4

There is also another important consistent check that needs to be verified here. Since we are working with very steep potentials, and consequently, also with very efficient and large dissipation, where $Q_{*} \gg 1$, one might worry how such a large dissipation might affect the spectrum beyond the linear order. Since in WI the inflaton and radiation perturbations get very strongly coupled at large dissipation ratios, we expect that large nonlinearities will emerge and adversely affect the spectrum of perturbations. As far the non-Gaussianities in WI are concerned, the Planck team has produced constraints for the so-called warm shape of the bispectrum in WI 68, with the non-Gaussianty coefficient denoted by $f_{\mathrm{NL}}^{\mathrm{warm}}$. In the analysis done in Ref. [68] and valid for the strong dissipative regime of WI (but for a temperature independent and constant dissipation ratio $Q$ ), the expression found for $f_{\mathrm{NL}}^{\mathrm{warm}}$, valid in the strong dissipative regime, was ${ }^{5}$ $f_{\mathrm{NL}}^{\text {warmS }}=-15 \ln (1+Q / 14)-5 / 2$. The Planck 2018 analysis 69] based on this expression has given the results, $f_{\mathrm{NL}}^{\mathrm{warmS}}=-48 \pm 27$ (from SMICA $+\mathrm{T}+\mathrm{E}, 68 \%$ C.L.) and $f_{\mathrm{NL}}^{\mathrm{warmS}}=-39 \pm 44$ (from SMICA $+\mathrm{T}, 68 \%$ C.L.), which then can be translated into the upper bounds for the dissipation ratio, $\log _{10} Q \leq 3.5$ and $\log _{10} Q \leq 3.6$, respectively, at $95 \%$ C.L. The largest values for $Q_{*}$ that we have and that are shown in Figs. 1 and 2 and to still to have a consistent value for $n_{s}, Q_{*} \lesssim 10^{3}$, all fall safely inside these upper bounds from the non-Gaussianity analysis. In particular, using the simple formula for the nonGaussianity parameter derived in Ref. 68 that was con-

3 This recent TCC condition was also recently analyzed in the context of WI in Ref. 66. However, we find that this conjecture (along with the older ones) requires in fact a much larger dissipation than the $Q \sim 20$ value estimated in Ref. 66. This is also backed up by the model studied in Ref. [47]. This is so because the conjecture TCC requires a very small scale for inflation in general and in WI this can only be achieved if very large $Q_{*}$ values are considered, at least for the presently known and treated models in the literature.

4 Note also that as recently claimed in Ref. [67], a larger scale for inflation than the one found in 65 might in fact be required from the TCC. Though this would lessen the condition on $Q$ here, we still need a sufficiently large $Q$ so as to have a temperature low enough at the end of inflation to satisfy the other bounds on the model.

5 Note that in the early Ref. 68 and also in the Planck papers on the non-Gaussianities, the notation used for the dissipation coefficient $Q$ was $r_{d}$. sidered by the Planck team, we find $\left|f_{\mathrm{NL}}^{\text {warm }}\right| \simeq 61.5$, when using the parameters of the explicit numerical example given above, with $Q_{*}=700$. Later on, in Ref. [70], the authors generalized the computation of $f_{\mathrm{NL}}^{\mathrm{warm}}$ for a temperature dependent $Q$ (and it was also pointed a sign error in the previous work). In Ref. [71], the authors made use of the $\delta N$ formalism to study the nonGaussianity. However, the $\delta N$ formalism better probes the non-Gaussianity of the local shape, while the warm shape of WI, valid in the strong dissipative regime, is very weakly correlated with the local shape [72. In Ref. [72], a complete analysis of non-Gaussianity in WI was performed, including the full effect of the coupling of the inflaton and radiation perturbations, which is essential to gauge the effect of the large dissipation on the spectrum. Following the numerical procedure explained in Ref. 72 and using the parameters values we have for the cubic form of the dissipation coefficient used in this work and the numerical example given above, for $Q_{*}=700$, we find the result $\left|f_{\mathrm{NL}}^{\mathrm{warm}}\right| \simeq 5.5$, which is small enough to satisfy the Planck bounds, but still large enough to possibly be probed in the future through more precise observations from both fourth generation $\mathrm{CMB}$ observatories and on also future large scale structure surveys, which are expected to bring down the present upper bounds on non-Gaussianities.

\section{CONCLUSIONS}

In this work, we have shown that dissipation effects, in the WI context, assist to achieve a consistent inflationary model with an exponential potential in the RS II braneworld. It also allows us to simultaneously satisfy all the theoretical and observational restrictions given previously on these type of exponential inflation potential in the braneworld construction. In fact, this is the first model of warm inflation with the specific form of a cubic temperature dependent dissipation coefficient that is found to be consistent with the observations in the strong dissipative regime of WI.

Achieving the strong dissipative regime in the present model is a consequence of both the braneworld high energy change of the GR evolution, combined with the exponential form for the primordial inflaton potential. As the result of achieving such a consistent dynamics in the strong dissipative regime, where $Q \gg 1$, it turns out that one can easily satisfy all the swampland conditions that have been recently proposed. Moreover, the model has a graceful exit from inflation for a large value of the constant parameter $\alpha$ in the inflaton exponential potential, with the dissipation ratio $Q$ decreasing and the brane correction disappearing at the end of inflation. Furthermore, although the brane correction can make the tensorto-scalar ratio $r$ larger in the high energy level, due to the change in the tensor spectrum, the dissipation effects suppress the energy scale of inflation more significantly in the strong dissipative regime and the model turns out to 
be fully consistent with observational data, with appropriate values for the spectral tilt and a highly suppressed tensor-to-scalar ratio, which is one of the main results of the present work. Additionally, the temperature of the Universe at the end of inflation is inside the range that the model does not suffer from potential gravitino overproduction and we can also avoid a large amount of relic gravity waves. We also note that these results follow for a broad range of the model parameter values, like for the constant $\alpha$ in the inflaton potential and for the brane tension $\lambda$, yet always satisfying the constrain bounds for these parameters. As also seen from the results shown in Figs. 1 and 2, there is a broad range of values for the dissipation coefficient that can be found to be consistent with the Planck data. In this sense, there is no special imposition or need for specific fine-tunings on these parameters. Likewise, as we study a regime of strong dissipation in WI, the inflationary slow-roll trajectory can be approached much faster, which reinforces the attractor like behavior of the corresponding slow-roll solution (see, e.g., Refs. 73, 74 for earlier studies of the effect of dissipation on the inflationary slow-roll trajectories).

We recall that exponential type of potentials have been considered as a possible candidate for describing the latetime acceleration [23, 75] observed in the recent Universe. However, by having a tracking behavior, it fails to properly act in the present-time as dark energy, with an equation of state that must be $\omega_{\phi} \simeq-1$. Besides, even in the context of the braneworld scenario, problems with this type of potential has been exposed [28], like a too large tensor-to-scalar ratio, a too red-tilted spectral index, and a possible excess of gravitational waves production that can destroy the BBN. From the results we have presented in this work, we see that all these problems can be overcome. Furthermore, typical interactions in the dark sector recently proposed in Ref. [36], and fully motivated from WI, could also be used in the present context. Since the Universe can smoothly enter into the radiation dominated epoch due to the presence of the WI dissipative effects, the reheating regime can be evaded, and the remaining inflaton field can be thawed and can behave like quintessence at latetime, like in the models studied in Ref. 36. Hence, the exponential potential can still be practical for unifying both the recent and the very early Universe histories, in the so-called dissipative quintessential inflation scenario. Therefore, our model can be a first step towards reconciling inflation and dark energy, with the bonus of being consistent with a high-energy UV completion within a theory of quantum gravity. We will study this possibility in the near future as a separate work.

\section{ACKNOWLEDGMENTS}

V.K's research at McGill has been supported by a NSERC Discovery Grant to Robert Brandenberger. R.O.R. is partially supported by research grants from Conselho Nacional de Desenvolvimento Científico e Tecnológico (CNPq), Grant No. 302545/2017-4, and Fundação Carlos Chagas Filho de Amparo à Pesquisa do Estado do Rio de Janeiro (FAPERJ), Grant No. E26/202.892/2017.
[1] N. Aghanim et al. (Planck Collaboration), Planck 2018 results. VI. Cosmological parameters, arXiv:1807.06209.

[2] J. Khoury, B. A. Ovrut, P. J. Steinhardt, and N. Turok, The ekpyrotic universe: Colliding branes and the origin of the hot big bang, Phys. Rev. D 64, 123522 (2001), arXiv:hep-th/0103239 P. J. Steinhardt, N. Turok, and N. Turok, A cyclic model of the universe, Science 296, 1436 (2002), arXiv:hep-th/0111030.

[3] F. Finelli and R. Brandenberger, On the generation of a scale invariant spectrum of adiabatic fluctuations in cosmological models with a contracting phase, Phys. Rev. D 65, 103522 (2002), arXiv:hep-th/0112249

[4] M. Gasperini and G. Veneziano, Pre-big bang in string cosmology, Astropart. Phys. 1, 317 (1993), arXiv:hepth/9211021

[5] A. Nayeri, R. H. Brandenberger, and C. Vafa, Producing a Scale-Invariant Spectrum of Perturbations in a Hagedorn Phase of String Cosmology, Phys. Rev. Lett. 97, 021302 (2006), arXiv:hep-th/0511140. R. H. Brandenberger, A. Nayeri, S. P. Patil, and C. Vafa, Tensor Modes from a Primordial Hagedorn Phase of String Cosmology, Phys. Rev. Lett. 98, 231302 (2007), arXiv:hepth/0604126

[6] K. Hinterbichler and J. Khoury, The pseudo-conformal Universe: Scale invariance from spontaneous breaking of conformal symmetry, J. Cosmol. Astropart. Phys. 04 (2012) 023, arXiv:1106.1428

[7] A. A. Starobinsky, A new type of isotropic cosmological models without singularity, Phys. Lett. 91B, 99 (1980); K. Sato, First order phase transition of a vacuum and expansion of the universe, Mon. Not. R. Astron. Soc. 195, 467 (1981); A. H. Guth, The inflationary Universe: A possible solution to the horizon and flatness problems, Phys. Rev. D 23, 347 (1981); A. Albrecht and P. J. Steinhardt, Cosmology for Grand Unified Theories with Radiatively Induced Symmetry Breaking, Phys. Rev. Lett. 48, 1220 (1982); A. D. Linde, A new inflationary Universe scenario: A possible solution of the horizon, flatness, homogeneity, isotropy and primordial monopole problems, Phys. Lett. 108B, 389 (1982).

[8] F. C. Adams, K. Freese, and A. H. Guth, Constraints on the scalar field potential in inflationary models, Phys. Rev. D 43, 965 (1991).

[9] A. Ijjas, P. J. Steinhardt, and A. Loeb, Inflationary paradigm in trouble after Planck2013, Phys. Lett. B 723, 261 (2013), arXiv:1304.2785

[10] J. Martin and R. H. Brandenberger, The TransPlanckian problem of inflationary cosmology, Phys. Rev. D 63, 123501 (2001), arXiv:hep-th/0005209 
[11] G. W. Gibbons and N. Turok, The measure problem in cosmology, Phys. Rev. D 77, 063516 (2008), arXiv:hepth/0609095

[12] A. Borde and A. Vilenkin, Eternal Inflation and The Initial Singularity, Phys. Rev. Lett. 72, 3305 (1994), arXiv:gr-qc/9312022

[13] S. Kachru, R. Kallosh, A. D. Linde, J. M. Maldacena, L. P. McAllister, and S. P. Trivedi, Towards inflation in string theory, J. Cosmol. Astropart. Phys. 10, (2003) 013, arXiv:hep-th/0308055 S. Kachru, R. Kallosh, A. D. Linde, and S. P. Trivedi, De Sitter vacua in string theory, Phys. Rev. D 68, 046005 (2003), arXiv:hepth/0301240

[14] G. Obied, H. Ooguri, L. Spodyneiko, and C. Vafa, De Sitter space and the Swampland, arXiv:1806.08362. $\mathrm{H}$. Ooguri and C. Vafa, On the geometry of the string landscape and the Swampland, Nucl. Phys. B766, 21 (2007), arXiv:hep-th/0605264 H. Ooguri, E. Palti, G. Shiu, and C. Vafa, Distance and de Sitter conjectures on the Swampland, Phys. Lett. B 788, 180 (2019), arXiv:1810.05506.

[15] E. Palti, The Swampland: Introduction and review, Fortsch. Phys. 67, 1900037 (2019), arXiv:1903.06239.

[16] R. Kallosh and A. Linde, Universality class in conformal inflation, J. Cosmol. Astropart. Phys. 07 (2013) 002, arXiv:1306.5220, R. Kallosh, A. Linde, and D. Roest, Superconformal inflationary $\alpha$-attractors, J. High Energy Phys. 11 (2013) 198, arXiv:1311.0472.

[17] H. C. Kim, G. Shiu, and C. Vafa, Branes and the Swampland, Phys. Rev. D 100, 066006 (2019), arXiv:1905.08261.

[18] L. Randall and R. Sundrum, An Alternative to Compactification, Phys. Rev. Lett. 83, 4690 (1999), arXiv:hepth/9906064 L. Randall and R. Sundrum, A Large Mass Hierarchy from a Small Extra Dimension, Phys. Rev. Lett. 83, 3370 (1999), arXiv:hep-ph/9905221

[19] P. Binetruy, C. Deffayet, and D. Langlois, Nonconventional cosmology from a brane universe, Nucl. Phys. B565, 269 (2000), arXiv:hep-th/9905012 P. Binetruy, C. Deffayet, U. Ellwanger, and D. Langlois, Brane cosmological evolution in a bulk with cosmological constant, Phys. Lett. B 477, 285 (2000), arXiv:hep-th/9910219. J. M. Cline, C. Grojean, and G. Servant, Cosmological Expansion in the Presence of Extra Dimensions, Phys. Rev. Lett. 83, 4245 (1999), arXiv:hep-ph/9906523.

[20] R. Maartens, D. Wands, B. A. Bassett, and I. Heard, Chaotic inflation on the brane, Phys. Rev. D 62, 041301 (2000), arXiv:hep-ph/9912464

[21] C. M. Lin, K. W. Ng, and K. Cheung, Chaotic inflation on the brane and the Swampland Criteria, Phys. Rev. D 100, 023545 (2019), arXiv:1810.01644.

[22] D. J. Gross and J. H. Sloan, The quartic effective action for the heterotic string, Nucl. Phys. B291, 41 (1987).

[23] E. J. Copeland, A. R. Liddle, and D. Wands, Exponential potentials and cosmological scaling solutions, Phys. Rev. D 57, 4686 (1998), arXiv:gr-qc/9711068 T. Barreiro, E. J. Copeland, and N. J. Nunes, Quintessence arising from exponential potentials, Phys. Rev. D 61, 127301 (2000), arXiv:astro-ph/9910214.

[24] F. Lucchin and S. Matarrese, Power law inflation, Phys. Rev. D 32, 1316 (1985).

[25] J. Yokoyama and K. i. Maeda, On the dynamics of the power law inflation due to an exponential potential, Phys. Lett. B 207, 31 (1988); A. R. Liddle, Power law infla- tion with exponential potentials, Phys. Lett. B 220, 502 (1989).

[26] E. J. Copeland, A. R. Liddle, and J. E. Lidsey, Steep inflation: Ending brane world inflation by gravitational particle production, Phys. Rev. D 64, 023509 (2001), arXiv:astro-ph/0006421

[27] V. Sahni, M. Sami, and T. Souradeep, Relic gravity waves from brane world inflation, Phys. Rev. D 65, 023518 (2001), arXiv:gr-qc/0105121.

[28] M. Dias and A. R. Liddle, On the possibility of braneworld quintessential inflation, Phys. Rev. D 81, 083515 (2010), arXiv:1002.3703

[29] A. Berera and L. Z. Fang, Thermally Induced Density Perturbations in the Inflation Era, Phys. Rev. Lett. 74, 1912 (1995), arXiv:astro-ph/9501024 A. Berera, Warm Inflation, Phys. Rev. Lett. 75, 3218 (1995), arXiv:astroph/9509049 A. Berera, M. Gleiser, and R. O. Ramos, A First Principles Warm Inflation Model that Solves the Cosmological Horizon / Flatness Problems, Phys. Rev. Lett. 83, 264 (1999), arXiv:hep-ph/9809583

[30] A. Berera, Interpolating the stage of exponential expansion in the early universe: A possible alternative with no reheating, Phys. Rev. D 55, 3346 (1997), arXiv:hep$\mathrm{ph} / 9612239$.

[31] A. Berera, T. W. Kephart, and S. D. Wick, GUT cosmic magnetic fields in a warm inflationary universe, Phys. Rev. D 59, 043510 (1999), arXiv:hep-ph/9809404

[32] R. H. Brandenberger and M. Yamaguchi, Spontaneous baryogenesis in warm inflation, Phys. Rev. D 68, 023505 (2003), arXiv:hep-ph/0301270 M. Bastero-Gil, A. Berera, R. O. Ramos, and J. G. Rosa, Warm baryogenesis, Phys. Lett. B 712, 425 (2012), arXiv:1110.3971. M. Bastero-Gil, A. Berera, R. O. Ramos, and J. G. Rosa, Observational implications of mattergenesis during inflation, J. Cosmol. Astropart. Phys. 10 (2014) 053, arXiv:1404.4976

[33] J. G. Rosa and L. B. Ventura, Warm Little Inflaton becomes Cold Dark Matter, Phys. Rev. Lett. 122, 161301 (2019), arXiv:1811.05493

[34] K. Dimopoulos and L. Donaldson-Wood, Warm quintessential inflation, Phys. Lett. B 796, 26 (2019), arXiv:1906.09648

[35] J. G. Rosa and L. B. Ventura, Warm little inflaton becomes dark energy, Phys. Lett. B 798, 134984 (2019), arXiv:1906.11835

[36] G. B. F. Lima and R. O. Ramos, Unified early and late Universe cosmology through dissipative effects in steep quintessential inflation potential models, Phys. Rev. D 100, 123529 (2019), arXiv:1910.05185

[37] A. Berera, I. G. Moss, and R. O. Ramos, Warm inflation and its microphysical basis, Rep. Prog. Phys. 72, 026901 (2009), arXiv:0808.1855

[38] S. Bartrum, M. Bastero-Gil, A. Berera, R. Cerezo, R. O. Ramos, and J. G. Rosa, The importance of being warm (during inflation), Phys. Lett. B 732, 116 (2014), arXiv:1307.5868

[39] L. M. H. Hall, I. G. Moss, and A. Berera, Scalar perturbation spectra from warm inflation, Phys. Rev. D 69, 083525 (2004), arXiv:astro-ph/0305015 L. Visinelli, Cosmological perturbations for an inflaton field coupled to radiation, J. Cosmol. Astropart. Phys. 01 (2015) 005, arXiv:1410.1187

[40] C. Graham and I. G. Moss, Density fluctuations from warm inflation, J. Cosmol. Astropart. Phys. 07 
(2009) 013, arXiv:0905.3500 M. Bastero-Gil, A. Berera, I. G. Moss, and R. O. Ramos, Cosmological fluctuations of a random field and radiation fluid, J. Cosmol. Astropart. Phys. 05 (2014) 004, arXiv:1401.1149. R. O. Ramos and L. A. da Silva, Power spectrum for inflation models with quantum and thermal noises, J. Cosmol. Astropart. Phys. 03 (2013) 032, arXiv:1302.3544. M. Bastero-Gil, A. Berera, and R. O. Ramos, Shear viscous effects on the primordial power spectrum from warm inflation, J. Cosmol. Astropart. Phys. 07 (2011) 030, arXiv:1106.0701.

[41] M. Benetti and R. O. Ramos, Warm inflation dissipative effects: Predictions and constraints from the Planck data, Phys. Rev. D 95, 023517 (2017), arXiv:1610.08758.

[42] M. Bastero-Gil and A. Berera, Warm inflation model building, Int. J. Mod. Phys. A 24, 2207 (2009), arXiv:0902.0521; S. Bartrum, M. Bastero-Gil, A. Berera, J. Mabillard, M. Pieroni, and R. O. Ramos, Identifying universality in warm inflation, J. Cosmol. Astropart. Phys. 07 (2018) 021, arXiv:1803.04982 L. L. Graef and R. O. Ramos, Probability of warm inflation in loop quantum cosmology, Phys. Rev. D 98, 023531 (2018), arXiv:1805.05985, M. Bastero-Gil, A. Berera, R. Hernandez-Jimenez, and J. G. Rosa, Dynamical and observational constraints on the Warm Little Inflaton scenario, Phys. Rev. D 98, 083502 (2018) arXiv:1805.07186, M. Bastero-Gil, S. Bhattacharya, K. Dutta, and M. R. Gangopadhyay, Constraining warm inflation with $C M B$ data, J. Cosmol. Astropart. Phys. 02 (2018) 054, arXiv:1710.10008; R. Arya, A. Dasgupta, G. Goswami, J. Prasad, and R. Rangarajan, Revisiting $C M B$ constraints on warm inflation, J. Cosmol. Astropart. Phys. 02 (2018) 043, arXiv:1710.11109. M. Motaharfar, E. Massaeli, and H. R. Sepangi, Power spectra in warm G-inflation and its consistency: Stochastic approach, Phys. Rev. D 96, 103541 (2017), arXiv:1705.04049, M. Motaharfar, E. Massaeli, and H. R. Sepangi, Warm Higgs $G$ inflation: Predictions and constraints from Planck 2015 likelihood, J. Cosmol. Astropart. Phys. 10 (2018) 002, arXiv:1807.09548 N. Videla and G. Panotopoulos, Observational constraints on warm quasi-exponential inflation, Phys. Rev. D 97, 123503 (2018), arXiv:1710.05046. Y. y. Wang, J. Y. Zhu and X. M. Zhang, Observational constraints on two-field warm inflation, Phys. Rev. D 99, 103529 (2019), arXiv:1905.02414

[43] I. Dymnikova and M. Khlopov, Self-consistent initial conditions in inflationary cosmology, Gravit. Cosmol. Suppl. 4, 50 (1998); I. Dymnikova and M. Khlopov, Decay of cosmological constant as Bose condensate evaporation, Mod. Phys. Lett. A 15, 2305 (2000), arXiv:astroph/0102094. I. Dymnikova and M. Khlopov, Decay of cosmological constant in selfconsistent inflation, Eur. Phys. J. C 20, 139 (2001).

[44] R. Herrera, N. Videla, and M. Olivares, Warm G inflation: Intermediate model, Phys. Rev. D 100, 023529 (2019), arXiv:1811.05510, R. Herrera, Reconstructing warm inflation, Eur. Phys. J. C 78, 245 (2018), arXiv:1801.05138, R. Herrera, G-Warm inflation, J. Cosmol. Astropart. Phys. 05 (2017) 029, arXiv:1701.07934. R. Herrera, M. Olivares, and N. Videla, General dissipative coefficient in warm intermediate and logamediate inflation, Phys. Rev. D 88, 063535 (2013), arXiv:1310.0780.
[45] M. Bastero-Gil, A. Berera, R. O. Ramos, and J. G. Rosa, Warm Little Inflaton, Phys. Rev. Lett. 117, 151301 (2016), arXiv:1604.08838.

[46] M. Motaharfar, V. Kamali and R. O. Ramos, Warm inflation as a way out of the swampland, Phys. Rev. D 99, 063513 (2019), arXiv:1810.02816, S. Das, Warm inflation in the light of Swampland criteria, Phys. Rev. D 99, 063514 (2019), arXiv:1810.05038. M. Bastero-Gil, A. Berera, R. Hernndez-Jimnez, and J. G. Rosa, Warm inflation within a supersymmetric distributed mass model, Phys. Rev. D 99, 103520 (2019), arXiv:1812.07296

[47] M. Bastero-Gil, A. Berera, R. O. Ramos, and J. G. Rosa, Towards a reliable effective field theory of inflation, arXiv:1907.13410

[48] M. A. Cid, S. del Campo, and R. Herrera, Warm inflation on the brane, J. Cosmol. Astropart. Phys. 10 (2007) 005, arXiv:0710.3148, S. del Campo and R. Herrera, Warm inflation in the DGP brane-world model, Phys. Lett. B 653, 122 (2007), arXiv:0708.1460 R. Herrera, N. Videla, and M. Olivares, Warped DGP model in warm intermediate inflation with a general dissipative coefficient in light of BICEP2 and Planck results, Phys. Rev. D 90, 103502 (2014), arXiv:1410.3776 V. Kamali and M. R. Setare, Warm-viscous inflation model on the brane in light of Planck data, Classical Quantum Gravity 32, 235005 (2015); R. Herrera and E. San Martin, Warm-intermediate inflationary universes on the brane, Int. J. Mod. Phys. D 22, 1350008 (2013); R. Herrera, $\mathrm{N}$. Videla, and M. Olivares, Warm intermediate inflation in the RandallSundrum II model in the light of Planck 2015 and BICEP2 results: A general dissipative coefficient, Eur. Phys. J. C 75, 205 (2015), arXiv:1504.07476.

[49] T. Shiromizu, K. i. Maeda, and M. Sasaki, The Einstein equation on the 3-brane world, Phys. Rev. D 62, 024012 (2000), arXiv:gr-qc/9910076.

[50] M. Bastero-Gil, A. Berera, and R. O. Ramos, Dissipation coefficients from scalar and fermion quantum field interactions, J. Cosmol. Astropart. Phys. 09 (2011) 033, arXiv:1008.1929 M. Bastero-Gil, A. Berera, R. O. Ramos, and J. G. Rosa, General dissipation coefficient in low-temperature warm inflation, J. Cosmol. Astropart. Phys. 01 (2013) 016, arXiv:1207.0445

[51] Y. Zhang, Warm inflation with a general form of the dissipative coefficient, J. Cosmol. Astropart. Phys. 03 (2009) 023, arXiv:0903.0685.

[52] M. Gleiser and R. O. Ramos, Microphysical approach to nonequilibrium dynamics of quantum fields, Phys. Rev. D 50, 2441 (1994), arXiv:hep-ph/9311278.

[53] G. Hinshaw et al. (WMAP Collaboration), Nine-year Wilkinson microwave anisotropy probe (WMAP) observations: Cosmological parameter results, Astrophys. J. Suppl. 208, 19 (2013), arXiv:1212.5226

[54] P. A. R. Ade et al. (Planck Collaboration), Planck 2013 results. XXII. Constraints on inflation, Astron. Astrophys. 571, A22 (2014), arXiv:1303.5082.

[55] I. G. Moss and C. Xiong, On the consistency of warm inflation, J. Cosmol. Astropart. Phys. 11 (2008) 023, arXiv:0808.0261 S. del Campo, R. Herrera, D. Pavón, and J. R. Villanueva, On the consistency of warm inflation in the presence of viscosity, J. Cosmol. Astropart. Phys. 08 (2010) 002, arXiv:1007.0103 M. Bastero-Gil, A. Berera, R. Cerezo, R. O. Ramos, and G. S. Vicente, Stability analysis for the background equations 
for inflation with dissipation and in a viscous radiation bath, J. Cosmol. Astropart. Phys. 11 (2012) 042, arXiv:1209.0712.

[56] C. D. Hoyle, U. Schmidt, B. R. Heckel, E. G. Adelberger, J. H. Gundlach, D. J. Kapner, and H. E. Swanson, Submillimeter Tests of the Gravitational Inverse Square Law: A Search for 'Large' Extra Dimensions, Phys. Rev. Lett. 86, 1418 (2001), arXiv:hep-ph/0011014.

[57] M. Kawasaki, K. Kohri, and T. Moroi, Hadronic decay of late-decaying particles and big-bang nucleosynthesis, Phys. Lett. B 625, 7 (2005), arXiv:astro-ph/0402490

[58] E. J. Copeland and O. Seto, Reheating and gravitino production in braneworld inflation, Phys. Rev. D 72, 023506 (2005), arXiv:hep-ph/0505149

[59] P. A. R. Ade et al. (Planck Collaboration), Planck 2015 results. XIV. Dark energy and modified gravity, Astron. Astrophys. 594, A14 (2016), arXiv:1502.01590

[60] V. Kamali, Reheating after Swampland conjecture, J. High Energy Phys. 01 (2020) 092, arXiv:1902.00701

[61] X. B. Li, H. Wang, and J. Y. Zhu, Gravitational waves from warm inflation, Phys. Rev. D 97, 063516 (2018), arXiv:1803.10074.

[62] D. Langlois, R. Maartens, and D. Wands, Gravitational waves from inflation on the brane, Phys. Lett. B 489, 259 (2000), arXiv:hep-th/0006007.

[63] Y. Akrami et al. (Planck Collaboration), Planck 2018 results. X. Constraints on inflation, arXiv:1807.06211.

[64] A. R. Liddle and S. M. Leach, How long before the end of inflation were observable perturbations produced?, Phys. Rev. D 68, 103503 (2003), arXiv:astro-ph/0305263.

[65] A. Bedroya and C. Vafa, Trans-Planckian Censorship and the Swampland, arXiv:1909.11063; A. Bedroya, R. Brandenberger, M. Loverde, and C. Vafa, TransPlanckian Censorship and inflationary cosmology, arXiv:1909.11106, S. Brahma, Trans-Planckian censorship, inflation and excited initial states for perturbations,
arXiv:1910.04741

[66] S. Das, Distance, de Sitter and trans-Planckian censorship conjectures: The status quo of warm inflation, Phys. Dark Univ. 27, 100432 (2020), arXiv:1910.02147

[67] S. Mizuno, S. Mukohyama, S. Pi and Y. L. Zhang, Universal upper bound on the inflationary energy scale from the trans-Planckian censorship conjecture, arXiv:1910.02979

[68] I. G. Moss and C. Xiong, Non-Gaussianity in fluctuations from warm inflation, J. Cosmol. Astropart. Phys. 04 (2007) 007, arXiv:astro-ph/0701302.

[69] Y. Akrami et al. (Planck Collaboration), Planck 2018 results. IX. Constraints on primordial non-Gaussianity, arXiv:1905.05697

[70] I. G. Moss and T. Yeomans, Non-Gaussianity in the strong regime of warm inflation, J. Cosmol. Astropart. Phys. 08 (2011) 009, arXiv:1102.2833.

[71] X. M. Zhang, H. Y. Ma, P. C. Chu, J. T. Liu, and J. Y. Zhu, Primordial non-Gaussianity in warm inflation using $\delta N$ formalism, J. Cosmol. Astropart. Phys. 03 (2016) 059, arXiv:1510.03138 X. M. Zhang, H. Y. Ma, P. C. Chu, and J. Y. Zhu, Primordial nonGaussianity in noncanonical warm inflation: Three- and four-point correlations, Phys. Rev. D 96, 043516 (2017), arXiv:1707.09154

[72] M. Bastero-Gil, A. Berera, I. G. Moss, and R. O. Ramos, Theory of non-Gaussianity in warm inflation, J. Cosmol. Astropart. Phys. 12 (2014) 008, arXiv:1408.4391.

[73] H. P. de Oliveira and R. O. Ramos, Dynamical system analysis for inflation with dissipation, Phys. Rev. D 57, 741 (1998), arXiv:gr-qc/9710093.

[74] R. O. Ramos, Fine tuning solution for hybrid inflation in dissipative chaotic dynamics, Phys. Rev. D 64, 123510 (2001), arXiv:astro-ph/0104379.

[75] U. França and R. Rosenfeld, Fine tuning in quintessence models with exponential potentials, J. High Energy Phys. 10 (2002) 015, arXiv:astro-ph/0206194. 